\title{
Dynamical properties of a Soliton in a Potential Well
}

\author{
B. Piette*and W.J. Zakrzewski ${ }^{\dagger}$ \\ Department of Mathematical Sciences, University of Durham, \\ Durham DH1 3LE, UK
}

October 2006

\begin{abstract}
We analyse the scattering of a two-dimensional soliton on a potential well. We show that this soliton can pass through the well, bounce back or become trapped and we study the dependence of the critical velocity on the width and the depth of the well. We also present a model based on a pseudo-geodesic approximation to the full system which shows that the vibrational modes of the soliton play a crucial role in the dynamical properties of its interactions with potential wells.
\end{abstract}

\section{Introduction}

The scattering of a soliton with a well is an interesting phenomenon for several reasons. First of all, it is an example of the dynamical evolution of a soliton in an inhomogeneous medium, in which case the parameters of the model are functions of space. As is well known, when waves scatter on a well, they can be partly reflected and partly transmitted. For solitons, the situation is more complicated as solitons cannot split and thus must either bounce, pass through or become trapped inside the well. This behaviour is very sensitive to the value of all the parameters of the model as well as to the initial conditions for the scattering.

The scattering of a soliton on a well is also interesting from a purely mathematical point of view. Very little is known about the general dynamical properties of non-integrable systems. Though many models have been studied numerically a general understanding

*e-mail address: B.M.A.G.Piette@durham.ac.uk

$\dagger^{\dagger}$ e-mail address: W.J.Zakrzewski@durham.ac.uk 
the vibrational modes of solitons play an important role [1] 2] and we shall show that the scattering of a soliton on a well is yet another example where this is indeed the case.

The scattering of solitons on potential obstructions has also been studied in many other papers. In particular, we have found a paper by Fei et al [3] who studied the scattering of one dimensional lattice Sine-Gordon kinks on a point defect. They found an interesting dependence on the initial kink velocity. Their work was taken further by Goodman and Haberman [4] who explained some of the results of [3] by introducing a low dimensional dynamical model involving the kink parameters together with a variable describing the amplitude of the standing wave localised around the defect. We have also looked at the scattering of Sine-Gordon kinks on potential obstructions (wells and barriers) and we are planning to report our results soon.

In a recent paper written in collaboration with Dr. Brand [5], we presented some results of our first study of the scattering of a two-dimensional soliton on a well of finite depth and width. We studied this scattering in a Baby Skyrme model whose solitons are often called skyrmions. There we showed that a soliton of this model can scatter through the well or become trapped, depending on its initial speed. In this paper, we analyse this scattering in more detail using numerical simulations and we also present a model based on a pseudo-geodesic approximation to the full system to explain our numerical results.

In the first section, we present the results of our numerical studies of the scattering of a soliton on a well, in which we varied both the width and the depth of the well. In the second section, we present two models based on pseudo-geodesic approximations to the full system and then use them to describe the scattering of the soliton with the well. We show that the vibrations of the soliton play a crucial role in its dynamical properties.

\section{$2 \quad$ Skyrme Model with a Potential Well}

In this paper we present the results of our study of the so called New Baby Skyrme model (NBS) in $2+1$ dimensions [6], in which the mass coefficient depends on the space variable $x$ so that it describes a well of width $L$, centred around $x=0$ and running parallel to the $y$ axis. The field of the model takes values on a 2 dimensional sphere $S^{2}$ and so can be described by a 3 component vector $\vec{\phi}=\left(\phi_{0}, \phi_{1}, \phi_{2}\right)$ of unit length : $|\vec{\phi}|=1$. The Lagrangian of the model is given by

$$
L=\frac{1}{2} \phi_{\mu}^{2}-\frac{\lambda}{4}\left(\phi_{\mu} \times \phi_{\nu}\right)^{2}-\frac{\lambda(1+\alpha)}{2}\left(1-\phi_{0}^{2}\right)
$$

where we have used the covariant notation $\phi_{\mu}^{2}=\phi_{t}^{2}-\phi_{x}^{2}-\phi_{y}^{2}$ and where

$$
\begin{array}{lll}
\alpha=a & & -L / 2<x<L / 2 \\
\alpha=0 & & \text { elsewhere }
\end{array}
$$


obstruction. When $a>0$ we have a potential barrier and the parameter $a$ describes its height; when $a$ is negative, we have a well and $|a|$ describes the depth of the well. Notice that $a \geq-1$. Given the interesting results in the well case that were reported in [5], in this paper we consider the case $a<0$.

Outside the well, $\alpha=0$, and so the parameters of the Skyrme and potential terms are identical. In this parametrisation, also used in [2], the parameter $\lambda$ is dimensionless and hence the soliton size varies with $\lambda$ only through its shape. When the parameters of the two terms in the Lagrangian differ, as is inside the well, one can rescale the space coordinates $x, y$ and the model parameters to make them equal. Calling $\lambda_{1}=\lambda$, the coefficient of the Skyrme term, and $\lambda_{2}=\lambda(1+a)$, the coefficient of the potential term, we perform the scaling

$$
\begin{aligned}
x_{i} & =\sigma X_{i} \quad i=1,2, x=x_{1}, \quad y=x_{2}, \\
\tilde{\lambda}_{1} & =\frac{1}{\sigma^{2}} \lambda_{1}, \\
\tilde{\lambda}_{2} & =\sigma^{2} \lambda_{2}
\end{aligned}
$$

and we see that if we choose $\sigma=\left(\lambda_{1} / \lambda_{2}\right)^{1 / 4}$ then $\tilde{\lambda}_{1}=\tilde{\lambda}_{2}=\left(\lambda_{1} \lambda_{2}\right)^{1 / 2}$ are the parameters of the model in dimensionless units.

Inside the well, $a<0$, and we have $\lambda_{1}=\lambda, \lambda_{2}=\lambda(1+a)$; so after the scaling we thus have

$$
\begin{aligned}
& \lambda_{e f}=\lambda(1+\alpha)^{1 / 2} . \\
& x_{e f}=x(1+\alpha)^{1 / 4} .
\end{aligned}
$$

We thus see that the effective value of the parameter, $\lambda_{e f f}$, is smaller inside the well. From this we can conclude that the energy of a static soliton inside the well is smaller than that of a soliton outside it. Moreover, the soliton is broader inside the well, as seen in Figure 1 where we have presented the plots of the energy profiles of the soliton for various well depths.

As the energy of a soliton is smaller inside the well, we see that the well attracts the soliton. To understand the scattering of the soliton on such a well, it is useful to have an idea of the energy of the soliton as a function of its position relative to the centre of the well. Such an energy profile is presented in figure 2 for various values of $L$ when $a=-0.8$. These energy profiles were obtained by solving numerically the time evolution of the soliton as its slides down into the well having added a friction term to the equations of motion to slow the soliton and absorb its kinetic energy. We see from figure 2 that due to the spatial extension of the soliton, the well is effectively smoothed out.

\subsection{Numerical Solutions}

As the Lagrangian (11) is invariant under Lorentz boosts along the $y$ direction we can, without any loss of generality, restrict ourselves to studying head on collisions of the 


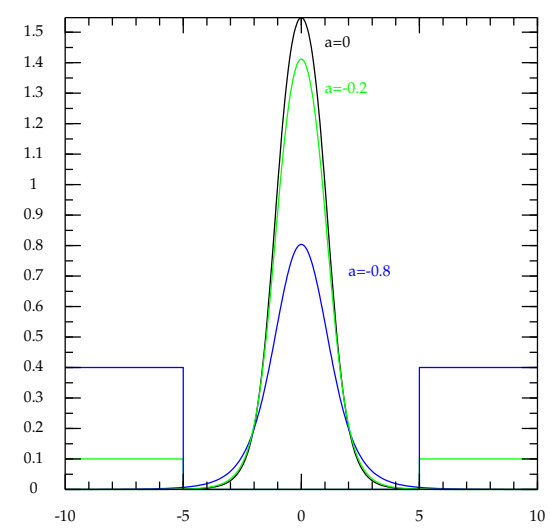

Figure 1: Energy profile of a soliton in the well for wells of width $L=10$ and depth $a=0, a=-0.2 \quad a=-0.8 . \lambda=0.5$.

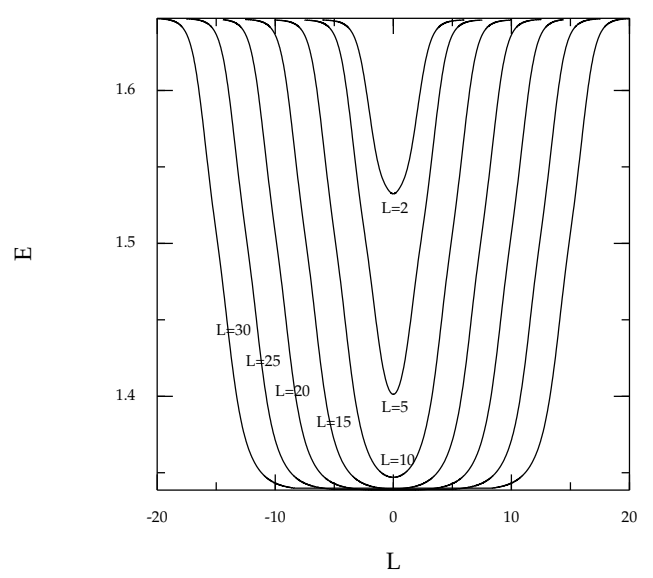

Figure 2: Potential energy of the soliton as a function of its position when $\lambda=0.5$. The well, of depth $a=-0.8$, is located between $x=-L / 2$ and $x=L / 2$. 
play any role as the well does not depend on that phase.

As reported in [5], the behaviour of the soliton when it scatters on the well depends very much on its initial speed. At very low speeds, the soliton falls into the well and gets trapped. For larger speeds, on the other hand, it passes through the well and emerges nearly unaltered (Fig. 3 d). For intermediate values of the speed, the trajectory of the soliton is extremely sensitive to its initial speed. In some instances, the soliton falls into the well, bounces against the opposite wall of the well, moves backwards and then escapes from the well in the direction it came from (Fig. 3] a). For other values of the speed, the soliton bounces twice inside the well before escaping from the well (Fig. B c). The ranges of initial speeds at which these different scatterings occur are very narrow and often are, maybe always, separated by regions where the soliton is trapped inside the well (Fig. 3 b). We suspect that the soliton can sometimes bounce more than twice inside the well, but due to the time required to perform the numerical simulations and the narrowness of the region where this could occur, we have not tried to find such a scattering.

As the soliton falls inside the well, it readjusts its shape and as a result it becomes excited i.e. it starts vibrating. The soliton of the NBS model has several vibrational modes with frequencies which depend on the parameter $\lambda$ of the model[2]. When the frequencies of oscillation of these vibrational modes are above the mass threshold, the vibrational modes are coupled to the radiation modes of the model resulting in a damping of the oscillations through radiation. When the frequencies are below the mass threshold, the soliton vibrations are stable, at least, in the linear approximation, when the amplitudes are sufficiently small.

In the NBS model there are two stable vibrational modes when $\lambda<1.1$. They correspond to the shape mode describing radial contraction and expansion, and the scattering mode involving alternating elongation and contraction along orthogonal directions. The frequencies of these two modes are very close to each other and they cross the mass threshold [2] around $\lambda \approx 0.27$.

Regardless of the value of $\lambda$, the soliton vibrations are always excited when it falls into the well and so some of the potential energy is always converted into the kinetic energy of these vibrations. This explains why, at small speeds, the soliton gets trapped inside the well. For large velocities, the kinetic energy of the translation is always large enough for the soliton to climb out of the well, and so the soliton just sails through it. In between these two extremes, the energy is transferred between the different degrees of freedom resulting in a complex dynamical system which we have to understand if we want to explain what we have seen in this process.

Very qualitatively, one can see that to escape from the well, the phases of the oscillations of the solitons must be such that the potential energy of the system is large enough just when the soliton gets to the edge of the well, while trying to climb out of it.

In figure 4 we present the outgoing speed of the soliton as a function of the incoming speed for two values of the parameter $\lambda$. One clearly sees that there is indeed a critical velocity below which the soliton can be trapped by the well. Moreover the outgoing speed 


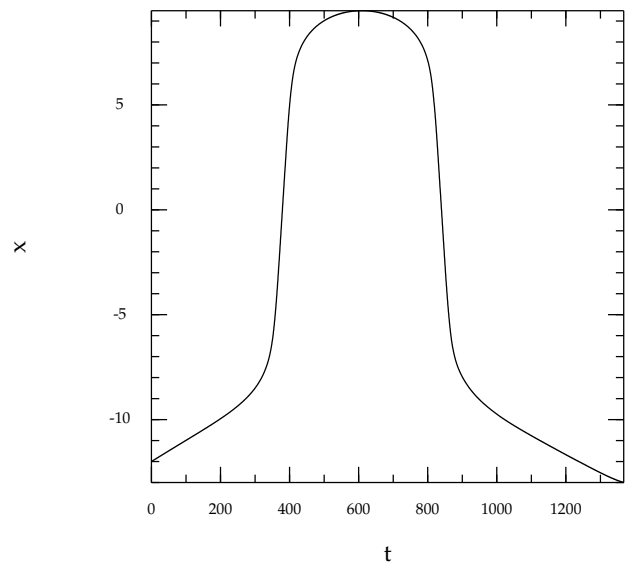

a

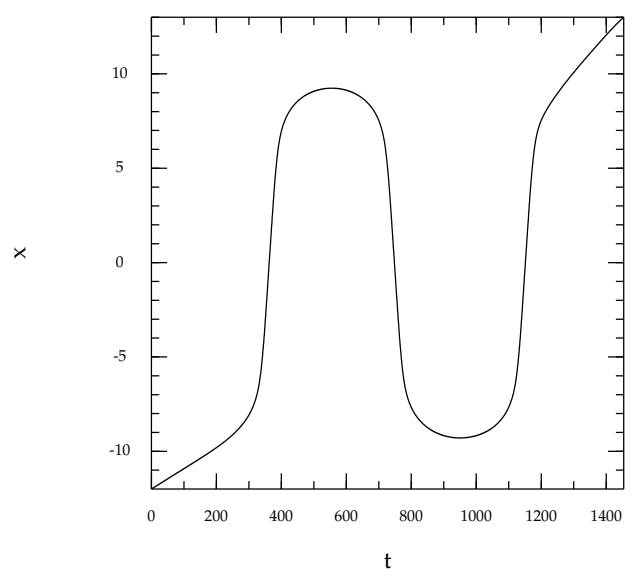

$\mathrm{C}$

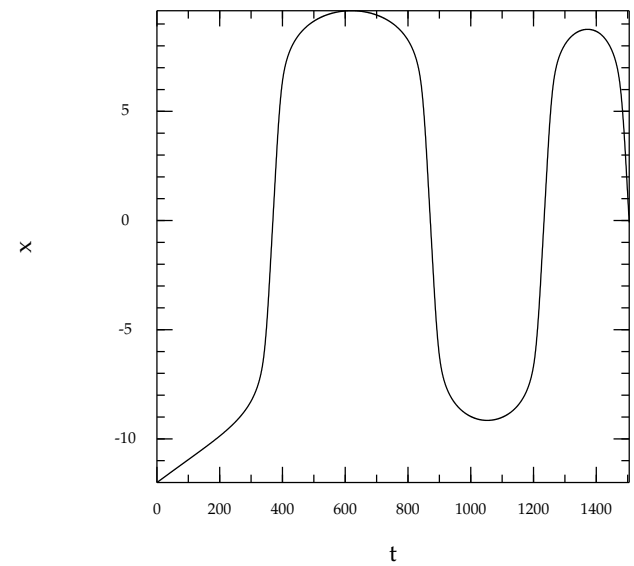

$\mathrm{b}$

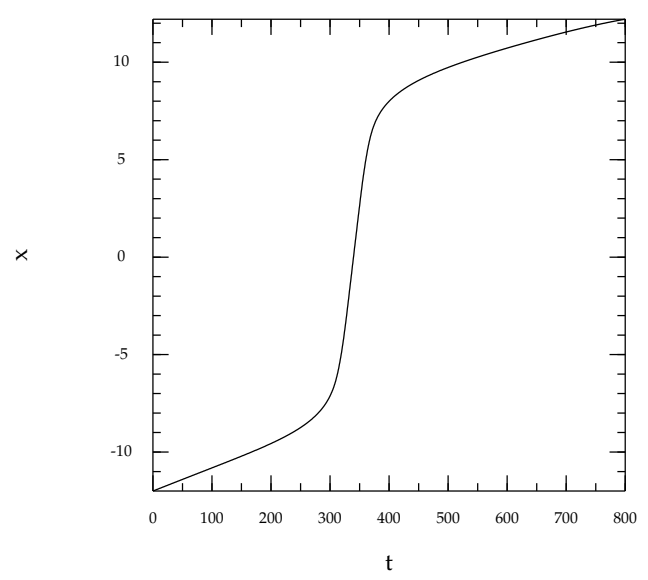

d

Figure 3: Trajectory of a soliton during the scattering for a well of width $L=10$ and depth $a=-0.2$ for $\lambda=0.5$ and a) $v=0.0102$, b) $v=0.0106$, c) $v=0.0109$, d) $v=0.012$. 


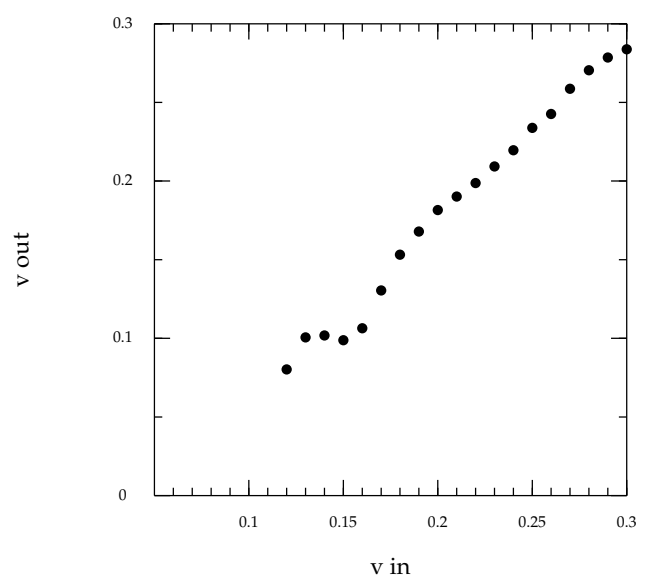

a

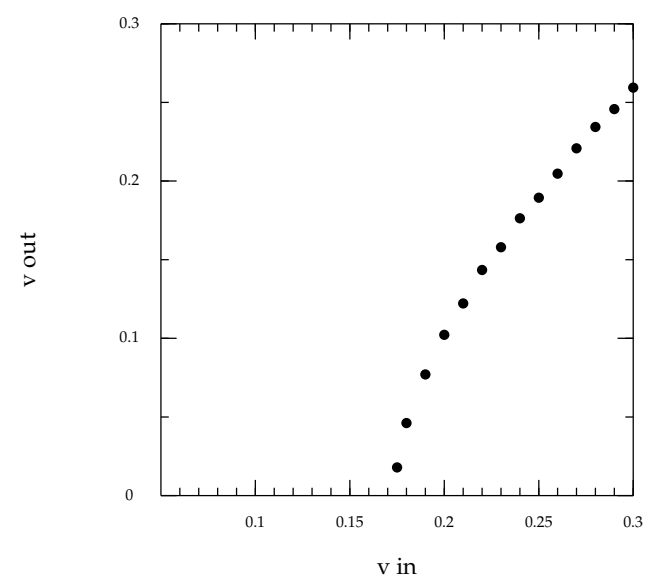

$\mathrm{b}$

Figure 4: Speed of the soliton after scattering on a well of width $L=10$ and depth $a=-0.8$ for a) $\lambda=0.2$. b) $\lambda=0.5$.

To analyse the scattering of the soliton on the well, we have computed the critical velocity of the soliton for different values of the parameters $\lambda$ and for different wells. In what follows, we define the critical speed as the speed above which the soliton is never trapped by the well. The results are shown in Figures [5:7.

From figure 5 we see that, as expected, the critical velocity increases when the well becomes deeper. The curve is relatively linear, but it exhibits a few humps. In the case $\lambda=0.2$ we also observe a plateau where $v_{c} \approx 0.05$ in the region $-0.5<a<0.05$. We believe this comes from the fact that every soliton vibrational mode radiates in this model and that even for a shallow well, the soliton looses some energy when it falls into the well, thus explaining the relatively large value of its critical speed.

In figure 6 we present the critical velocity as a function of the parameter $\lambda$ and we see that, at least for our choice of the well, the critical velocity increases with $\lambda$.

In figure 7 we present the dependence of the critical velocity on the width of the well $L$. When the well is narrower than the soliton itself, $L<10$, the critical velocity increases with $L$ except for a small hump in the region $3<L<5$. When $L>10$ the curve exhibits oscillations with very regular amplitudes and periods which depend on $\lambda$. These oscillations can be explained by the fact that to climb out of the well, the soliton must be in the correct phase of vibration. The phase is itself dictated by the frequency of vibration and the time needed to cross the well which is directly related to the speed of the soliton. So as the length of the well changes, the speed of the soliton must also change to allow it to escape, but if the length of the well is extended by the distance travelled by the soliton during one period of oscillation, then the critical speed returns to its previous value. 


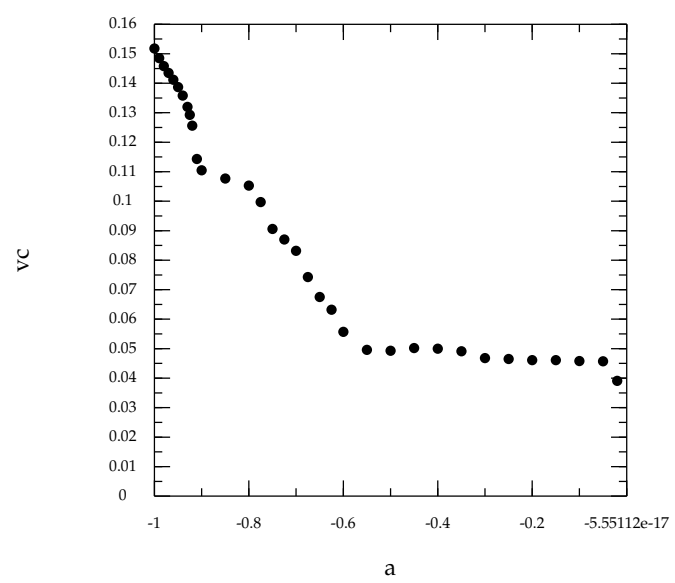

a

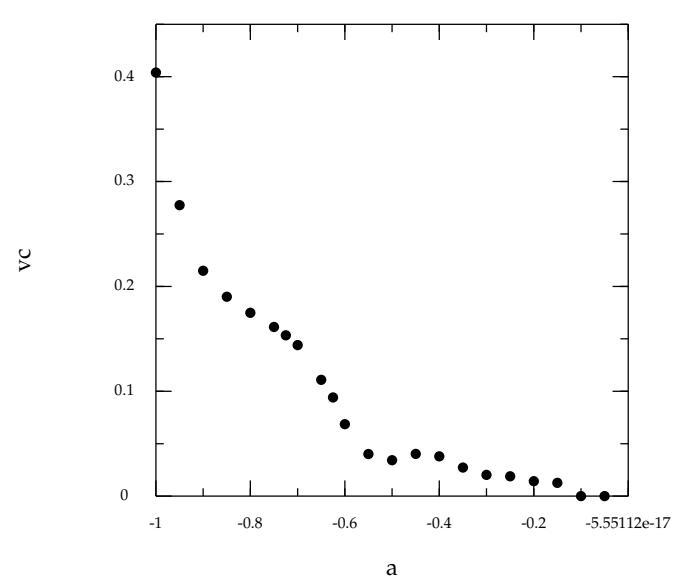

$\mathrm{b}$

Figure 5: Critical velocity as a function of the depth of the well $a$ for $L=10$ and a) $\lambda=0.2$. b) $\lambda=0.5$.

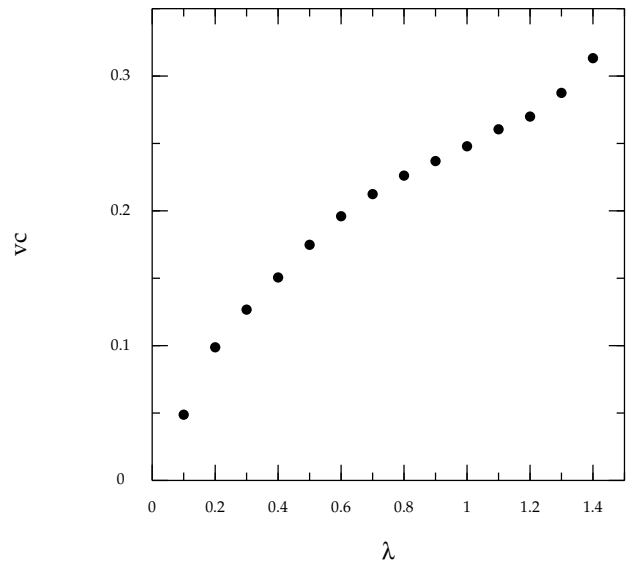

Figure 6: Critical velocity as a function of the parameter $\lambda$ for $L=10$ and $a=-0.8$. 


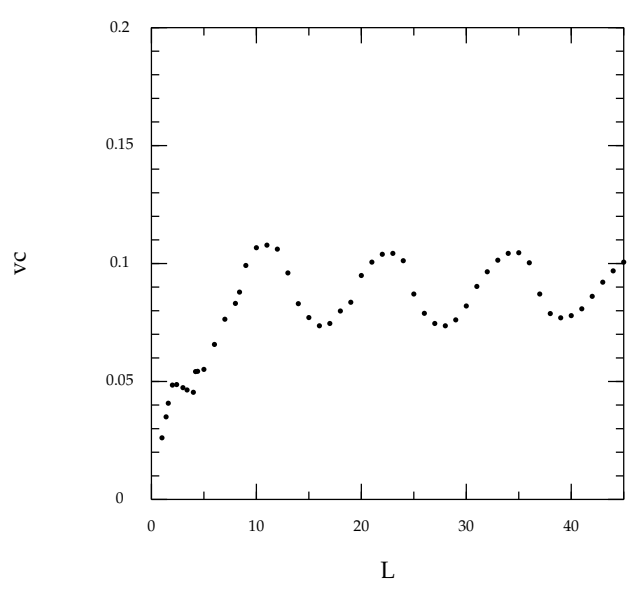

a

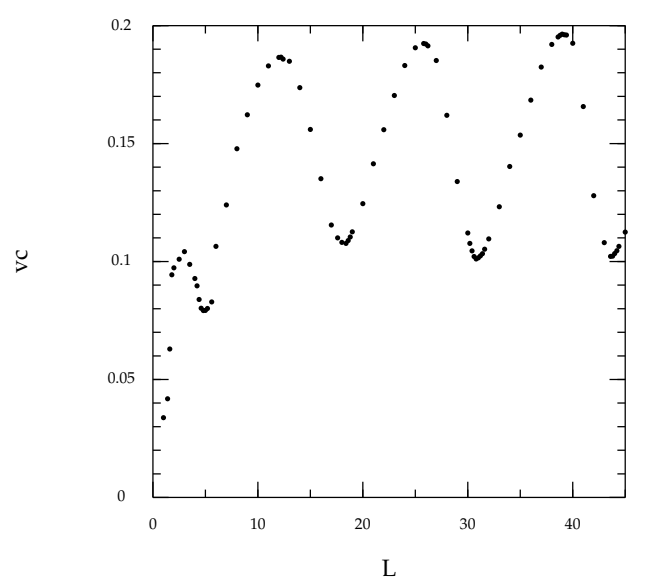

$\mathrm{b}$

Figure 7: Critical velocity as a function of the well width $L$ for $a=-0.8$ and a) $\lambda=0.2$. b) $\lambda=0.5$.

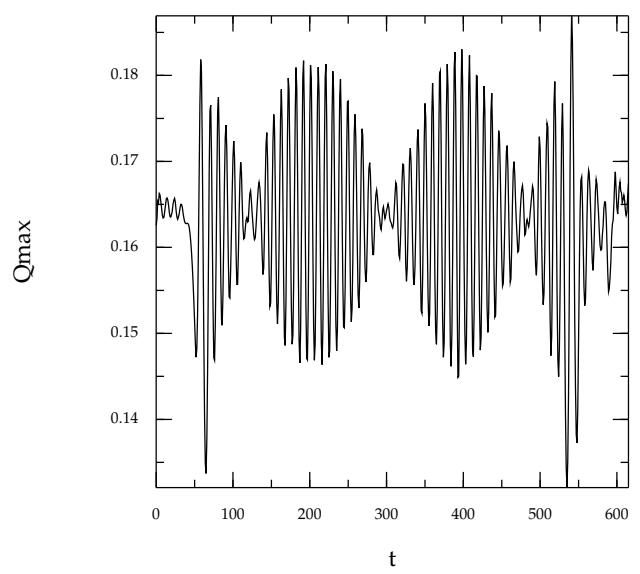

Figure 8: Oscillation of the topological charge density maximum during a one bounce scattering for $\lambda=0.5, L=2, a=-0.8$ and $v=0.0970313$. 
average speeds are, respectively, $v=0.09$ and $v=0.15$ and the periods of oscillation are thus given by $T=133$ and $T=87$, respectively. These periods are about 6 times larger than the frequency of the oscillation of the soliton [2]. So even though our explanation looks reasonable, the actual data do not support it however, looking at figure 8 we note that the oscillations of the maximum of the topological charge density show that several modes of oscillations are excited. Our original explanation has just been too naive and we need to take into account the role of several vibrational modes. Incidentally, Figure 8 corresponds to the case where the soliton bounces in the well once, around $t=300$ and then escapes from the well, around $t=600$, going back to where it came from.

In the next section we present a model, based on a pseudo-geodesic approximation. This model shows that, while our initial picture is essentially correct, some details of it need a modification. This is due to the fact that several degrees of freedom do indeed come into play in this process and so lead to a larger oscillation in $L$. This leads us to an improved pseudo-geodesic model which, qualitatively, correctly describes the scattering of the soliton with the well.

\subsection{Models based on a Pseudo Geodesic Approximation}

To explain the characteristics of the scattering of the NBS soliton with the well we present a model which is a generalisation of the geodesic approximation to the full process.

Note that have we had an analytical expression to describe the evolution of the soliton as it interacts with the well we would have approximated the soliton by a rigid object evolving in an effective potential induced by the well. To describe the soliton vibrations, we would have then to consider a small perturbation around this "gradient flow" and expand the Hamiltonian to quadratic order in this perturbation. This would have led us to a dynamical system with zero modes and vibrational modes identical to those of the solitons, at least in the small amplitude limit, which would evolve in an effective potential describing the changes experienced by the soliton as it falls into the well.

As we do not have an analytical expression for the time evolution of the soliton, we must thus adopt a different approach, and so we have decided to generalise the pseudogeodesic approximation introduced in [2, i.e. by trying to construct a dynamical system that has similar vibrational modes as the soliton and that evolves in a potential approximating the one experienced by the soliton falling into the well.

As the soliton falls into the well, effectively, it goes down a potential more or less described by Fig 2. To model the vibrations of the soliton, we have first tried to use a system made out of two masses separated by a finite distance, related to the size of the soliton, and linked together by a spring in such a way that the frequency of the normal mode of the system matches the main vibrational mode of the NBS soliton. Moreover, the distance between the masses and the spring tension have to be a function of the position of the centre of mass to model the parameter changes that occur as the soliton moves inside the well. 
introduce the profile

$$
P(s ; x)=\tanh (s(x-L / 2))+\tanh (s(-x-L / 2)) .
$$

where $s$ is a scale parameter which has to be fitted.

The potential in which the 2 masses evolve is then given by

$$
\begin{aligned}
V(x)= & E_{0}+\frac{d E}{2} c P(s ; x) \\
& c= \begin{cases}(L / 10)^{1 / 4} & L<10 \\
0 & L \geq 10\end{cases}
\end{aligned}
$$

where $E_{0}$ is the energy of the soliton outside the well and $E-d E$ is the energy of the soliton when it is at rest inside the well. The parameter $c$ is a factor that is required to correct the potential depth when the well is too narrow, i.e. when only a part of the soliton is located inside the well. The potential (77) constitutes a good approximation to the curves in figure 1 when one takes $s \approx 0.5$.

The equilibrium distance between the two masses should be a function of the position of the centre of mass, $x_{c m}=\frac{1}{2}\left(x_{1}+x_{2}\right)$, and so we set it to twice the radius of the soliton. Defining $D_{0}(\lambda)$ as the equilibrium distance outside the well, we choose the position dependant equilibrium distance as

$$
D\left(x_{c m}\right)=D_{0}-\frac{1}{2} d P\left(s_{D} ; x_{c m}\right),
$$

where $s_{D}$ is a scale parameter and $d$ is the difference between the equilibrium distance between the 2 masses inside and outside the well.

The Hamiltonian of our effective model is thus given by

$$
H=\frac{1}{2}\left(M{\dot{x_{1}}}^{2}+M{\dot{x_{2}}}^{2}\right)+\frac{k}{2}\left(x_{2}-x_{1}-D\left(x_{c m}\right)\right)^{2}+\frac{1}{2}\left(V\left(x_{1}\right)+V\left(x_{2}\right)\right)
$$

where $2 M=E_{0}$ is the energy of the soliton at rest and $k=M[0.3+0.63(1-\exp (-1.58 \lambda))]^{1 / 2}$ (cfr [2]) sets the vibration frequency of the soliton.

In Table 1 we give some of the numerical values that we have used for this pseudogeodesic approximation.

\begin{tabular}{|l|l|l|l|l|}
\hline & $E_{0}$ & $d E$ & $D_{0}$ & $d$ \\
\hline$\lambda=0.2, a=-0.8$ & 1.30701 & 0.149235 & 3.1611 & 1.73382 \\
$\lambda=0.2, a=-0.2$ & 1.30701 & 0.0267838 & 3.1611 & 0.19758 \\
$\lambda=0.5, a=-0.8$ & 1.64923 & 0.312953 & 3.05734 & 1.64716 \\
$\lambda=0.5, a=-0.2$ & 1.64923 & 0.0572152 & 3.05734 & 0.18602 \\
\hline
\end{tabular}

Table 1: Parameter values for the 2 mass pseudo-geodesic model 


$$
\begin{aligned}
& M \ddot{x_{1}}=-k\left(x_{2}-x_{1}-D\left(x_{c m}\right)\right)\left(-1+\frac{1}{4} d G\left(s_{D} ; x_{c m}\right)\right)-\frac{d E}{4} G\left(s ; x_{1}\right) \\
& M \ddot{x_{2}}=-k\left(x_{2}-x_{1}-D\left(x_{c m}\right)\right)\left(1+\frac{1}{4} d G\left(s_{D} ; x_{c m}\right)\right)-\frac{d E}{4} G\left(s ; x_{2}\right)
\end{aligned}
$$

where

$$
G(s ; x)=\frac{\partial P(s ; x)}{\partial x}=s\left(\tanh ^{2}(s(-x-L / 2))-\tanh ^{2}(s(x-L / 2))\right) .
$$

We can also add a friction term proportional to $\dot{x_{1}}-\dot{x_{2}}$ to model the radiation of the soliton. In the new baby Skyrme model, the vibrations of a single soliton are coupled to radiation when $\lambda<\lambda_{c}=0.27$. If we define

$$
\lambda_{e f f}=\lambda-\frac{1}{2} \lambda\left((1+a)^{1 / 2}-1\right) P\left(s_{D} ; x\right)
$$

we can then add the following friction force when $k_{e f f}<k_{c}$ :

$$
\begin{aligned}
& F r_{x_{1}}=-K_{f r}\left(\lambda_{c}-\lambda_{e f f}\right)\left(\dot{x_{1}}-\dot{x_{2}}\right) \\
& F r_{x_{2}}=-K_{f r}\left(\lambda_{c}-\lambda_{e f f}\right)\left(\dot{x_{2}}-\dot{x_{1}}\right)
\end{aligned}
$$

where $K_{f r}$ is a friction coefficient.

In figure 9 we present the outgoing velocity of the soliton as a function of the incoming speed obtained in the model based on the 2 mass pseudo-geodesic approximation for the same cases as those of figure 4 . We note that when the speed is small enough the soliton is trapped. Sometimes, however, the soliton bounces several times in the well and eventually escapes from the well even when the initial speed is below the critical velocity. This is particularly clear from fig 9.b where we see that the soliton can escape in any direction and with almost any speed that is restricted by the conservation of energy.

In figure 10 we plot the critical velocity as a function of the well width $L$. We observe oscillations like those in figure [7, with a similar amplitude, but the period of these oscillations is about 5 times too small. The oscillations can be explained by the fact that to escape from the well, the soliton must be in the correct phase. This phase depends on the time spent by the soliton in the well, which itself depends on the width of the well. If the critical velocity is 0.1 , and as the frequency of the oscillation is roughly 20 one would expect the period of oscillation to be around 2 in our dimensionless units, and this is what we see in figure 10. The soliton on the other hand oscillates with a period roughly 5 times larger (Fig. 7). This suggests that for the soliton, several excited oscillation modes contribute to the build up of the correct phase.

The NBS model, in fact, has several vibrational modes [2] and it is the superposition of all these modes that determines the phase of the total system. In a complex system like the soliton, the extrema of the critical velocity as a function of $L$ will be determined by how close or how far the system is from the ideal phase for achieving the escape from 


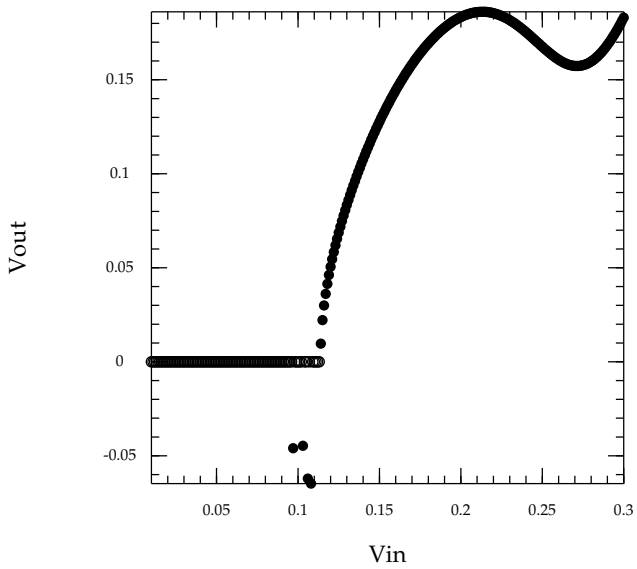

a

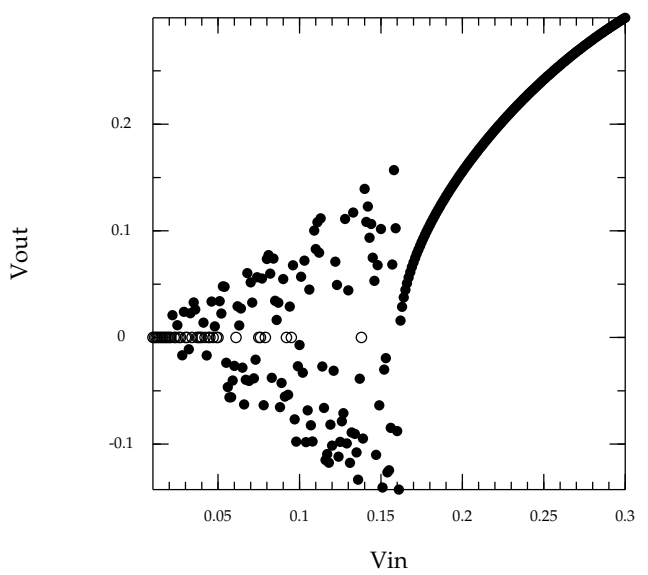

$\mathrm{b}$

Figure 9: Speed of a soliton in the 2 mass model after scattering on a well of width $L=10$ and depth $a=-0.8$ for a) $\lambda=0.2, s=s_{D}=1.6, K_{f r}=0.01$ b) $\lambda=0.5$, $s=0.5, s_{D}=1.6, K_{f r}=0$.

the well. The periodicity of $V_{c}(L)$ can thus differ from the exact periodicity of the full system, but nevertheless, the periods of oscillation of a system with several vibrational modes are usually larger than the lowest frequency, explaining why the oscillations in Fig (7) are larger than the frequency of the lowest vibrational mode, which our previous naive argument expected to match the period of $V_{c}(L)$.

To show that this is indeed the case we next consider a model based on a multi-mode pseudo-geodesic approximation.

\subsection{Multi-mode Pseudo-Geodesic Model}

The simplest model based on a multi-mode pseudo geodesic approximation would involve 3 masses linked by two identical springs. For symmetry reasons, the two external masses must be identical. If the frequency ratio of the two vibrational modes that we try to model is of the order of 10 percent then the two external masses must be about 10 times smaller than the central mass and as a result the system does not absorb enough energy to reproduce the scattering of a NBS soliton (the predicted critical velocity turns out to be much too small).

We thus need to construct a model with more degrees of freedom and we have chosen to use a system made out of 4 identical masses linked by 6 springs in total, as pictured in figure 12

Defining the vector $\vec{Z}_{i}=\left(x_{i}, y_{i}\right)$ and the distance $R_{i j}=\left|\vec{Z}_{i}-\vec{Z}_{j}\right|$ we see that the 


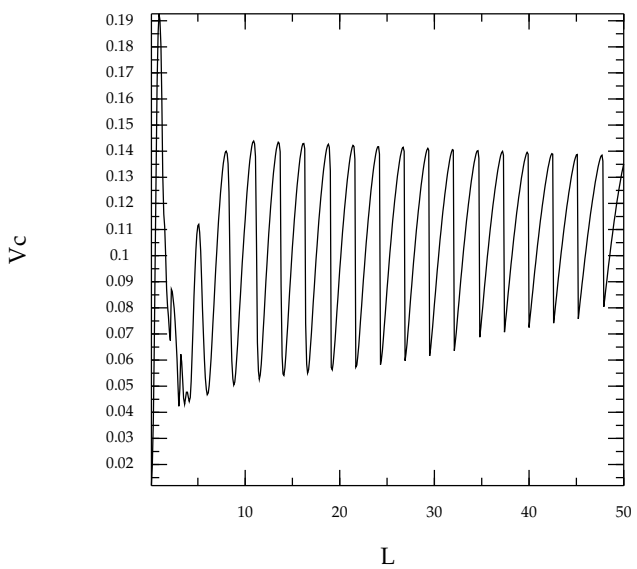

a

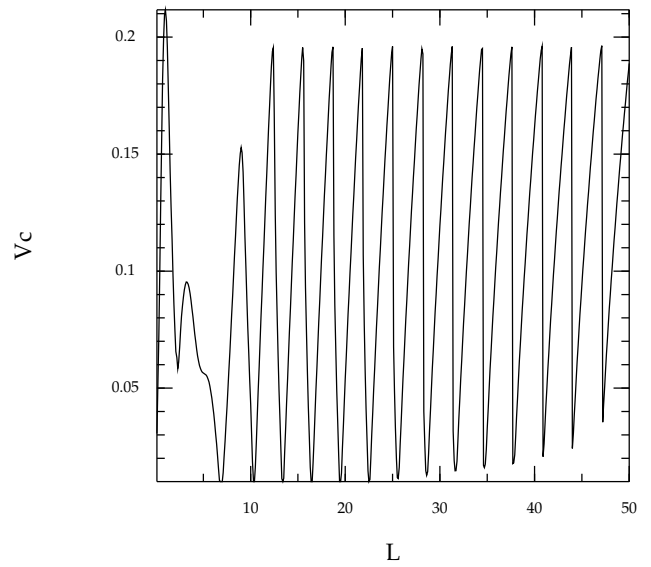

$\mathrm{b}$

Figure 10: Critical velocity in the 2 mass model as a function of the well width $L$ for $a=$ -0.8 and a) $\left.\lambda=0.2, s=s_{D}=1.6, K_{f r}=0.01 \mathrm{~b}\right) \lambda=0.5, s=1.05, s_{D}=1.05, K_{f r}=0$.

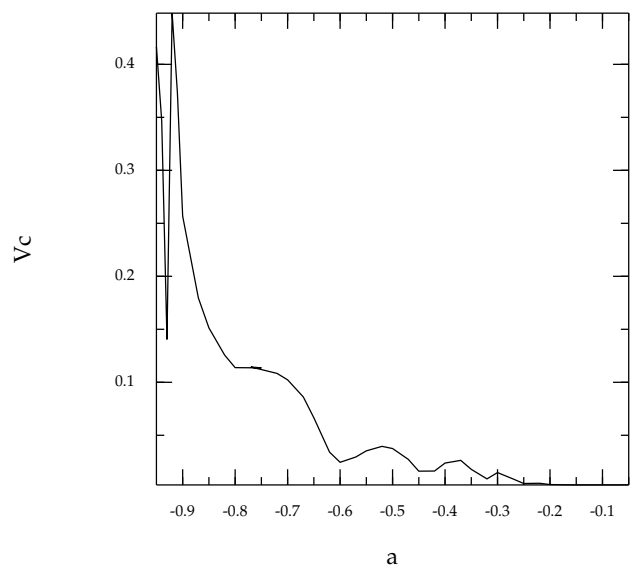

a

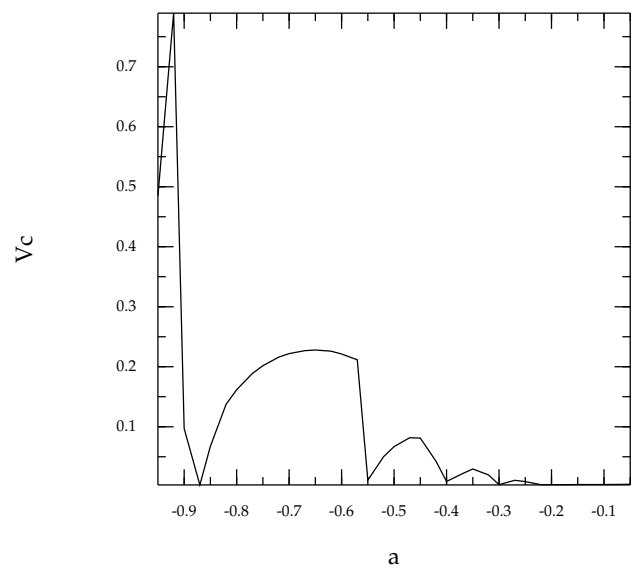

$\mathrm{b}$

Figure 11: Critical velocity in the 2 mass model as a function of the well depth $a$ for $L=10$ and a) $\lambda=0.2$. b) $\lambda=0.5$. 


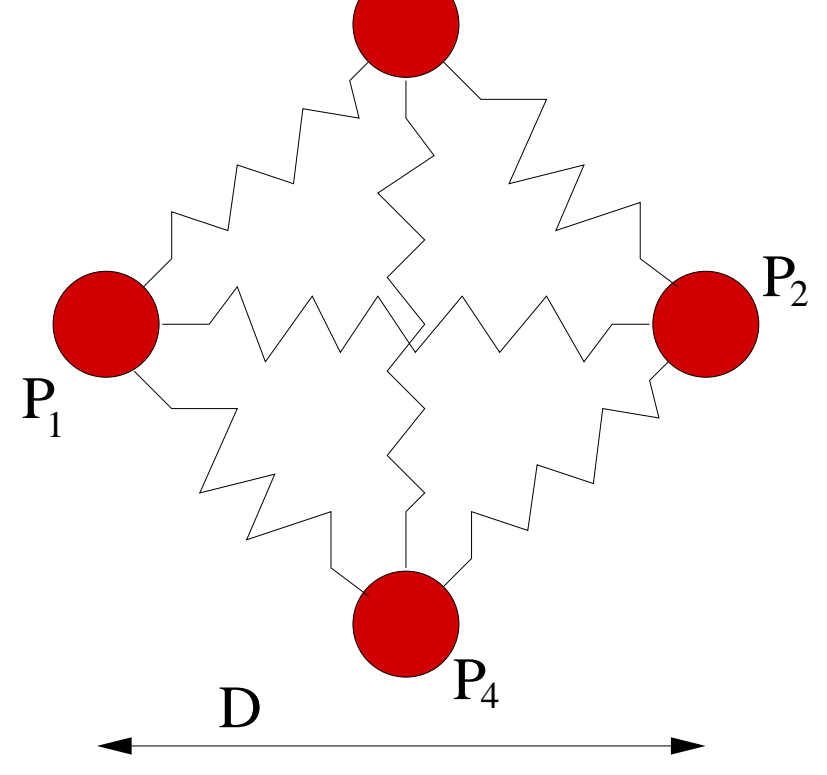

Figure 12: Configuration of the 4 mass model.

Hamiltonian of this system is given by:

$$
\begin{aligned}
H= & \frac{1}{2}\left(M{\dot{\overrightarrow{Z_{1}}}}^{2}+M{\dot{\overrightarrow{Z_{2}}}}^{2}+M{\dot{\overrightarrow{Z_{3}}}}^{2}+M \dot{\vec{Z}}_{4}^{2}\right)+\frac{k_{a}}{2}\left(\left(R_{12}-D\right)^{2}+\left(R_{34}-D\right)^{2}\right) \\
& +\frac{k_{b}}{2}\left(R_{13}-\frac{D}{\sqrt{2}}\right)^{2}+\frac{k_{b}}{2}\left(R_{14}-\frac{D}{\sqrt{2}}\right)^{2}+\frac{k_{b}}{2}\left(R_{23}-\frac{D}{\sqrt{2}}\right)^{2}+\frac{k_{b}}{2}\left(R_{24}-\frac{D}{\sqrt{2}}\right)^{2} \\
& +\frac{1}{2}\left(V\left(x_{1}\right)+V\left(x_{2}\right)+V\left(x_{3}\right)+V\left(x_{4}\right)\right) .
\end{aligned}
$$

Here $k_{a}$ and $k_{b}$ are the two parameters which determine the frequencies of the normal modes of the system and $V(x)$ is the potential (7). In addition, $D$ is the equilibrium distance between the 2 pairs of masses and is given by (17) where $x_{c m}=\left(x_{1}+x_{2}+x_{3}+x_{4}\right) / 4$. Moreover, $E_{0}=4 M$ so that the system has the same rest mass as our NBS soliton.

To see whether this model reproduces the results of our simulations of the NBS scatterings we must, first of all, compute the vibration frequencies of the system when the potential $V(x)=0$. This is required to determine the values of $k_{a}$ and $k_{b}$. To do this, we expand $Z_{i}$ around its equilibrium position $\bar{Z}_{i}: x_{i}=\bar{x}_{i}+d x_{i}$ and $y_{i}=\bar{y}_{i}+d y_{i}$. To quadratic order, the Hamiltonian (13) becomes

$$
\begin{aligned}
H_{0}= & M\left(\dot{d x_{1}}{ }^{2}+\dot{d y}_{1}{ }^{2}+\dot{d x}_{2}{ }^{2}+d \dot{y}_{2}{ }^{2}+\dot{d x}_{3}{ }^{2}+d \dot{y}_{3}{ }^{2}+d \dot{x}_{4}{ }^{2}+\dot{d y_{4}}{ }^{2}\right) \\
& +k_{a}\left(\left(d x_{1}-d x_{2}\right)^{2}+\left(d y_{3}-d y_{4}\right)^{2}\right) \\
& +k_{b}\left(\left(d x_{1}-d x_{3}+d y_{1}-d y_{3}\right)^{2}+\left(d x_{1}-d x_{4}-d y_{1}+d y_{4}\right)^{2}\right. \\
& \left.+\left(d x_{2}-d x_{3}-d y_{2}+d y_{3}\right)^{2}+\left(d x_{2}-d x_{4}+d y_{2}-d y_{4}\right)^{2}\right) .
\end{aligned}
$$

and so we see that the system posseses 5 vibrational modes and 3 null modes with the following frequencies and the corresponding eigenmodes:

- Breathing mode: $\omega_{b r}^{2}=4 k_{a} / M$; eigen vector: $x_{1}=-1, x_{2}=1, y_{3}=-1, y_{4}=1$. 
- Bend mode 1: $\omega_{b 1}^{2}=8 k_{b} / M$; eigen vector: $y_{1}=-1, y_{2}=-1, y_{3}=1, y_{4}=1$.

- Bend mode 2: $\omega_{b 2}^{2}=8 k_{b} / M$; eigen vector: $x_{1}=-1, x_{2}=-1, x_{3}=1, x_{4}=1$.

- Squeeze mode: $\omega_{s q}^{2}=8 k_{b} / M$; eigen vector: $y_{1}=1, y_{2}=-1, x_{3}=-1, x_{4}=1$.

- X translation2: $\omega_{t r x}^{2}=0$; eigen vector: $x_{1}=1, x_{2}=1, x_{3}=1, x_{4}=1$.

- Y translation: $\omega_{t r y}^{2}=0$; eigen vector: $y_{1}=1, y_{2}=1, y_{3}=1, y_{4}=1$.

- Rotation: $\omega_{\text {rot }}^{2}=0$; eigen vector: $y_{1}=-1, y_{2}=1, x_{3}=-1, x_{4}=1$.

To model the NBS solitons, we fix the parameters $k_{b}$ and $k_{a}$ by fitting $\omega_{b r}$ and $\omega_{s h}$ to the values of the corresponding frequencies of a NBS soliton: $\omega_{b r}=(0.3+$ $0.63(1-\exp (-1.58 \lambda)))^{1 / 2}$. Choosing $k_{b}=K_{a b} k_{a}$ we then take $k_{a}=\frac{M}{4}(0.3+0.63(1-$ $\exp (-1.58 \lambda)))^{1 / 2}$ and $\omega_{s h}=K_{\omega} \omega_{b r}$. Using the expressions for the normal modes $\omega_{s h}$ and $\omega_{b r}$ we have $K_{a b}=\left(k_{\omega}^{2}-1\right) / 2$.

Moreover, the effective value of $\lambda$ varies when the soliton falls into the whole. To take this effect into account, we introduce the profile

$$
\lambda_{\text {eff }}=\lambda\left(1-\frac{1}{2}\left((1+\alpha)^{1 / 2}-1\right) P\left(S_{\lambda} ; x_{c} m\right)\right)
$$

which we then use in the expression for $k_{a}$ :

$$
k_{a}=\frac{M}{4}\left(0.3+0.63\left(1-\exp \left(-1.58 \lambda_{e f f}\right)\right)\right)^{1 / 2},
$$

making it position dependant.

If we define

$Q=\frac{1}{8}\left(\left(R_{12}-D\right)^{2}+\left(R_{34}-D\right)^{2}+K_{a b}\left(\left(R_{13}-\frac{D}{\sqrt{2}}\right)^{2}+\left(R_{14}-\frac{D}{\sqrt{2}}\right)^{2}+\left(R_{23}-\frac{D}{\sqrt{2}}\right)^{2}+\left(R_{24}-\frac{D}{\sqrt{2}}\right)^{2}\right)\right) \frac{d k_{a}}{d x_{c m}}$

then the equations of motion for the degrees of freedom of our Hamiltonian (13) are given by

$$
\begin{aligned}
M \ddot{x_{1}}= & -k_{a}\left(R_{12}-D\right) \frac{\left(x_{1}-x_{2}\right)}{R_{12}}-k_{a}\left(R_{12}+R_{34}-2 D\right) \frac{1}{8} d G\left(s_{D} ; x_{c m}\right) \\
& -k_{b}\left(R_{13}-\frac{D}{\sqrt{2}}\right) \frac{\left(x_{1}-x_{3}\right)}{R_{13}}-k_{b}\left(R_{14}-\frac{D}{\sqrt{2}}\right) \frac{\left(x_{1}-x_{4}\right)}{R_{14}} \\
& -k_{b}\left(R_{13}+R_{14}+R_{23}+R_{24}-4 \frac{D}{\sqrt{2}}\right) \frac{1}{8} d G\left(s_{D} ; x_{c m}\right)-Q-\frac{d E}{4} G\left(s ; x_{1}\right) \\
M \ddot{y_{1}}= & -k_{a}\left(R_{12}-D\right) \frac{\left(y_{1}-y_{2}\right)}{R_{12}}-k_{b}\left(R_{13}-\frac{D}{\sqrt{2}}\right) \frac{\left(y_{1}-y_{3}\right)}{R_{13}}-k_{b}\left(R_{14}-\frac{D}{\sqrt{2}}\right) \frac{\left(y_{1}-y_{4}\right)}{R_{14}}
\end{aligned}
$$




$$
\begin{aligned}
M \ddot{x_{2}=} & -k_{a}\left(R_{12}-D\right) \frac{\left(x_{2}\right.}{R_{12}}-k_{a}\left(R_{12}+R_{34}-2 D\right) \frac{1}{8} d G\left(s_{D} ; x_{c m}\right) \\
& -k_{b}\left(R_{13}-\frac{D}{\sqrt{2}}\right) \frac{\left(x_{2}-x_{3}\right)}{R_{23}}-k_{b}\left(R_{14}-\frac{D}{\sqrt{2}}\right) \frac{\left(x_{2}-x_{4}\right)}{R_{24}} \\
& -k_{b}\left(R_{13}+R_{14}+R_{23}+R_{24}-4 \frac{D}{\sqrt{2}}\right) \frac{1}{8} d G\left(s_{D} ; x_{c m}\right)-Q-\frac{d E}{4} G\left(s ; x_{2}\right) \\
M \ddot{y_{2}=} & -k_{a}\left(R_{12}-D\right) \frac{\left(y_{2}-y_{1}\right)}{R_{12}}-k_{b}\left(R_{13}-\frac{D}{\sqrt{2}}\right) \frac{\left(y_{2}-y_{3}\right)}{R_{13}}-k_{b}\left(R_{14}-\frac{D}{\sqrt{2}}\right) \frac{\left(y_{2}-y_{4}\right)}{R_{14}} \\
M \ddot{x_{3}=} & -k_{a}\left(R_{34}-D\right)\left(\frac{\left(x_{3}-x_{4}\right)}{R_{34}}-k_{a}\left(R_{12}+R_{34}-2 D\right) \frac{1}{8} d G\left(s_{D} ; x_{c m}\right)\right. \\
& -k_{b}\left(R_{31}-\frac{D}{\sqrt{2}}\right) \frac{\left(x_{3}-x_{1}\right)}{R_{31}}-k_{b}\left(R_{32}-\frac{D}{\sqrt{2}}\right) \frac{\left(x_{3}-x_{2}\right)}{R_{32}} \\
& -k_{b}\left(R_{13}+R_{14}+R_{23}+R_{24}-4 \frac{D}{\sqrt{2}}\right) \frac{1}{8} d G\left(s_{D} ; x_{c m}\right)-Q-\frac{d E}{4} G\left(s ; x_{3}\right) \\
M \ddot{y_{3}}= & -k_{a}\left(R_{34}-D\right) \frac{\left(y_{3}-y_{4}\right)}{R_{34}}-k_{b}\left(R_{31}-\frac{D}{\sqrt{2}}\right) \frac{\left(y_{3}-y_{1}\right)}{R_{31}}-k_{b}\left(R_{32}-\frac{D}{\sqrt{2}}\right) \frac{\left(y_{3}-y_{2}\right)}{R_{32}} \\
M \ddot{x_{4}}= & -k_{a}\left(R_{34}-D\right) \frac{\left(x_{4}-x_{3}\right)}{R_{34}}-k_{a}\left(R_{12}+R_{34}-2 D\right) \frac{1}{8} d G\left(s_{D} ; x_{c m}\right) \\
& -k_{b}\left(R_{41}-\frac{D}{\sqrt{2}}\right) \frac{\left(x_{4}-x_{1}\right)}{R_{41}}-k_{b}\left(R_{42}-\frac{D}{\sqrt{2}}\right) \frac{\left(x_{4}-x_{2}\right)}{R_{42}} \\
& -k_{b}\left(R_{13}+R R_{14}+R_{23}+R_{24}-4 \frac{D}{\sqrt{2}}\right) \frac{1}{8} d G\left(s_{D} ; x_{c m}\right)-Q-\frac{d E}{4} G\left(s ; x_{4}\right) \\
M \ddot{y_{4}}= & -k_{a}\left(R_{34}-D\right) \frac{\left(y_{4}-y_{3}\right)}{R_{34}}-k_{b}\left(R_{41}-\frac{D}{\sqrt{2}}\right) \frac{\left(y_{4}-y_{1}\right)}{R_{41}}-k_{b}\left(R_{42}-\frac{D}{\sqrt{2}}\right) \frac{\left(y_{4}-y_{2}\right)}{R_{42}} .
\end{aligned}
$$

We could also add various friction terms but, to keep the model simple, we have just added twi single friction terms proportional to $\dot{x_{2}}-\dot{x_{1}}$ and $\dot{y_{3}}-\dot{y_{4}}$. We thus assume that only the diagonal springs can radiate. In the new baby Skyrme model, the lowest vibrational mode of a single soliton is coupled to the radiation when $\lambda<\lambda_{c_{\text {min }}}=0.27$. As $\lambda=w_{c}^{2}$, for higher vibration frequency the critical value of $\lambda$ is given by $\lambda_{c}=K_{\omega}^{2} \lambda_{c_{m i n}}$. We have thus added the following friction force when $\lambda_{\text {eff }}<\lambda_{c}$ :

$$
\begin{aligned}
& F r_{x_{1}}=-K_{f r}\left(\lambda_{c}-\lambda_{e f f}\right)\left(\dot{x_{1}}-\dot{x_{2}}\right) \\
& F r_{x_{2}}=-K_{f r}\left(\lambda_{c}-\lambda_{e f f}\right)\left(\dot{x_{2}}-\dot{x_{1}}\right) \\
& F r_{y_{3}}=-K_{f r}\left(\lambda_{c}-\lambda_{e f f}\right)\left(\dot{y_{3}}-\dot{y_{4}}\right) \\
& F r_{y_{4}}=-K_{f r}\left(\lambda_{c}-\lambda_{e f f}\right)\left(\dot{y_{4}}-\dot{y_{3}}\right),
\end{aligned}
$$

where $\lambda_{\text {eff }}$ is given by (11) and $K_{f r}$ is a friction coefficient. We have also tried to add some friction along the lateral strings, but we have found that this did not model well the dissipation of energy by the soliton so from now on we set such friction to zero.

Solving numerically the equations of motion (18) we found that the scale factor $s, s_{D}$ and $s_{\lambda}$ can be set to the same value, as one would expect. Trying to reproduce the data shown in Fig. [7from the full 2 dimensional equations, we have found that the best values of the model parameters are given by 
- $\lambda=0.5: s=s_{D}=s_{\lambda}=0.47, K_{\omega}=1.63$ and $K_{f r}=0.02$.

Figure 13 shows the dependence of the critical velocity as a function of the well width $L$ for the 4 mass model.

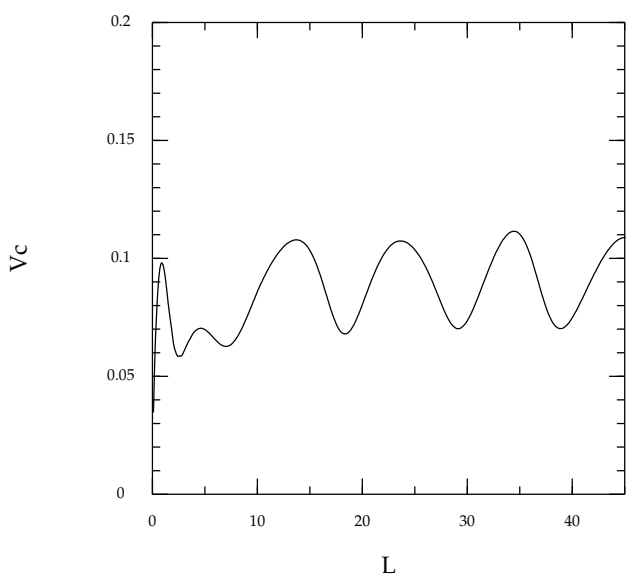

a

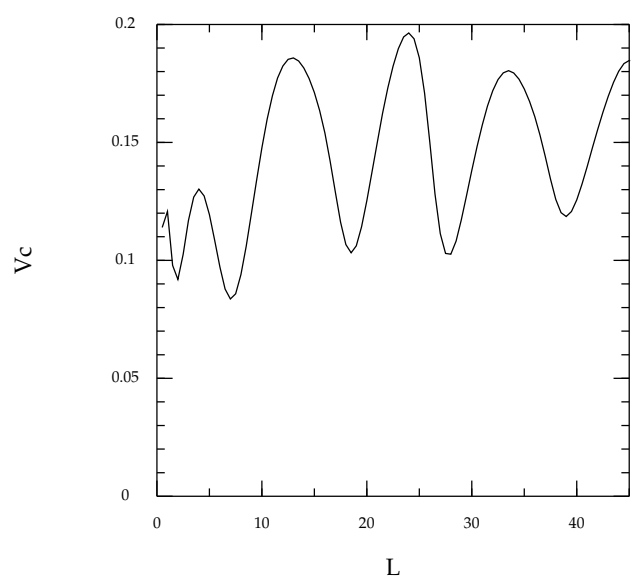

$\mathrm{b}$

Figure 13: Critical velocity in the 4 mass model as a function of the well width $L$ for $a=-0.8$ and a) $\lambda=0.2, s=s_{D}=s_{k}=0.4, K_{f r}=0.02, K_{\omega}=1.45$ b) $\lambda=0.5$, $s=s_{D}=s_{k}=0.47, K_{f r}=0.025, K_{\omega}=1.63$.

Like the 2 mass model, the 4 mass model predicts that the value of the critical velocity oscillates as the well width $L$ increases. The period of oscillations are nevertheless larger than in the 2 mass model and is controlled in our model mainly by the parameter $K_{\omega}$. The amplitude of oscillations and the minimum of the oscillations are on the other hand determined mostly by the parameters $s$ and $K_{f r}$. When $K_{f r}$ is small, the critical velocity is very small but the oscillations are then very irregular.

The 4 mass model reproduces the correct range of quantities describing the oscillation for both $\lambda=0.2$ and $\lambda=0.5$. Though the curves in Fig [13] and Fig [7] do not march perfectly, their general features are similar. Even the small hump just below $L=5$, is more or less reproduced.

Having set the parameters of the 4 mass model, we then obtained the figures 14 and [15] which should be compared with figures 4, and 5 respectively.

We notice in particular in Fig. 14 that the relation between the ingoing and outgoing velocity are very similar, even if the predicted critical velocity is slightly smaller than the real one. When $\lambda=0.2$ the outgoing velocity has a small dip around $v_{\text {in }}=0.15$, Fig $4 \mathrm{a}$, a feature that our 4 mass model does not reproduce. We believe that this is caused by a 


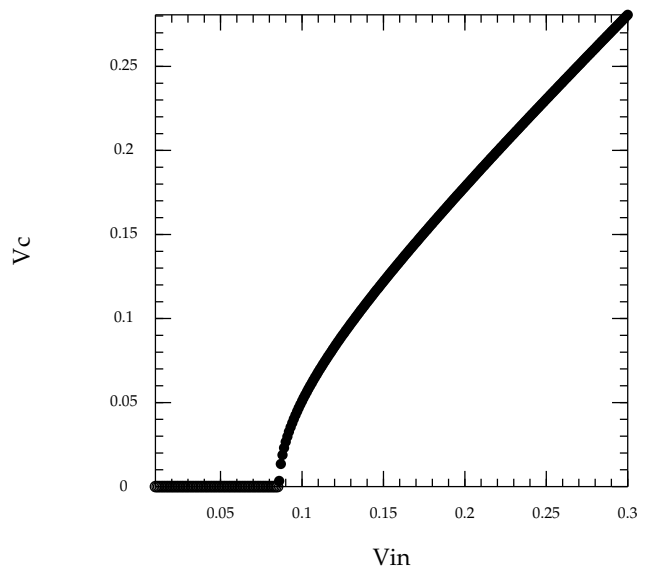

a

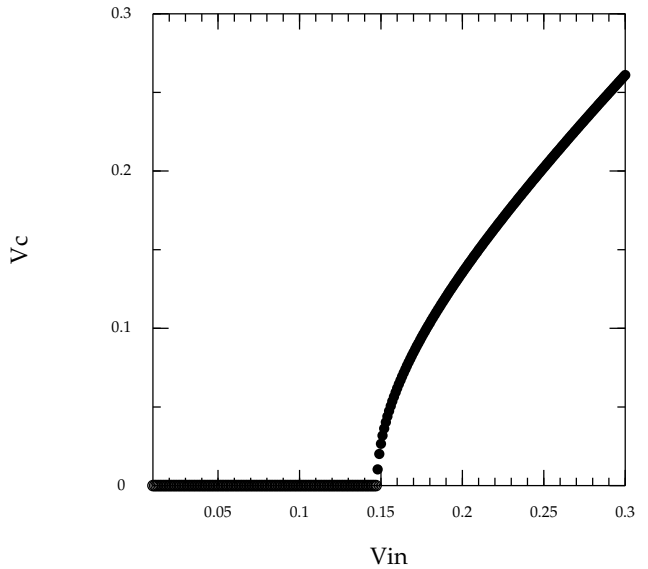

b

Figure 14: Speed of a soliton in the 4 mass model after scattering on a well of width $L=10$ and depth $a=-0.8$ for a) $\lambda=0.2$ b) $\lambda=0.5$. [To be compared with Fig. 4 ]

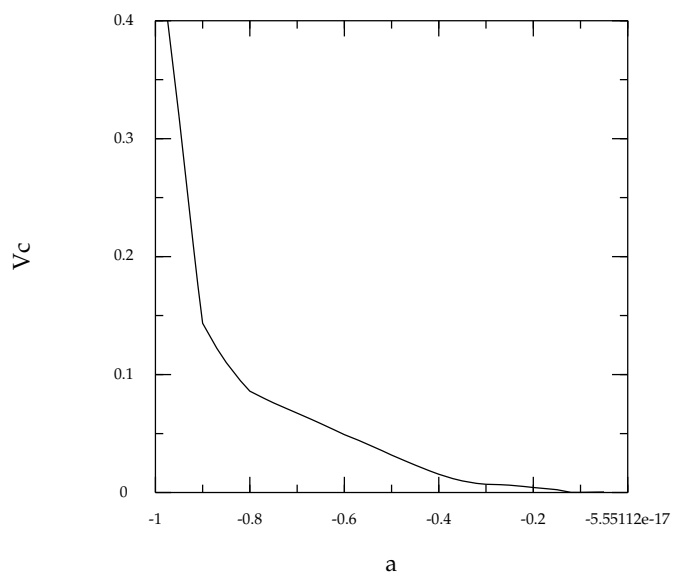

a

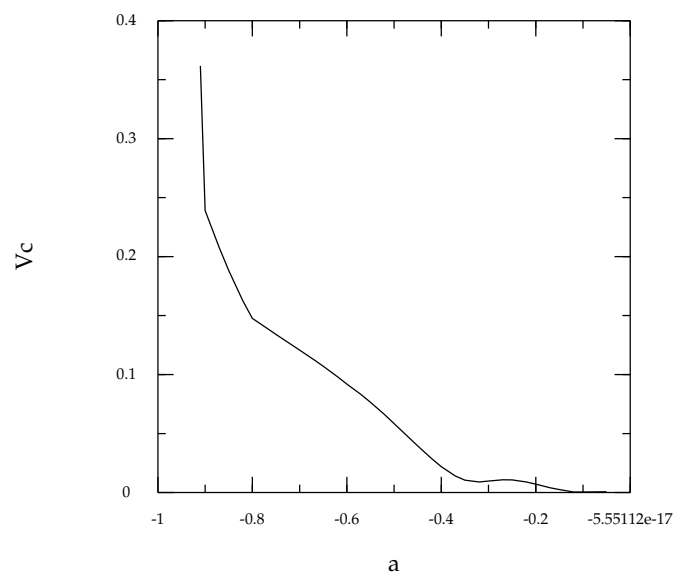

b

Figure 15: Critical velocity in the 4 mass model as a function of the well depth $a$ for $L=10$ and a) $\lambda=0.2$. b) $\lambda=0.5$. [To be compared with Fig. [5] 
Figure 15 presents the dependence of the critical velocity as a function of the depth of the well $a$. The model works surprisingly well when $\lambda=0.5$ reproducing the gross features of Figure 5 except when $a$ is close to -1 . In this region, the soliton is very broad when it is inside the well and so its overlap with the edges of the well is not properly taken into account by our pseudo-geodesic approximation.

When $\lambda=0.2$ the dependence of the critical speed on the parameter $a$ predicted by the pseudo-geodesic approximation is very crude. In particular, we do not reproduce the plateau where $V_{c}$ is constant for small values of $|a|$. We attribute this to the important role of the dispersion of energy through radiation that our pseudo-geodesic approximation takes into account only very crudely.

The fact that the four mass pseudo-geodesic approximation reproduces the properties of the NBS soliton much better than the 2 mass model shows very clearly that it is the interference between several vibrational modes of the soliton that determines the scattering properties of the soliton on the well. It is the relative phases between these vibrational modes that determine the variation of the critical velocity when the parameters of the model are changed.

The pseudo-geodesic approximation reproduces well the observation that when the soliton falls into the well it gets excited. A fraction of its kinetic energy is transferred to oscillation modes. To escape from the well, the soliton needs to be in a correct phase, meaning that all its oscillations must be in a correct phase. The fact that the parameter $K_{\omega}$ is larger than 1.1 shows that it is not just the two lowest vibrational modes that matter, but that the higher pseudo vibrational modes, which radiate, play a key role as well.

\section{Conclusion}

We have shown that the scattering of the NBS soliton on a well has several interesting properties. At large speeds, the soliton crosses the well nearly elastically, while at small speeds it is trapped by the well. For a very small range of values of the parameters, the soliton can sometimes bounce a few times inside the well before escaping from it.

The major factor in the interaction between the well and the soliton comes from the fact that the size of the soliton at rest inside the well is different from its size outside the well. Moreover, the energy of the soliton is smaller inside the well. As the soliton falls into the well, it alters its shape and as a result, its vibrational modes become exited. These oscillations absorb some energy from the soliton, making it impossible for the soliton to escape from the well unless it has enough kinetic energy to make up for the amount of energy stored in the vibrational modes. 
tional modes that get excited during the scattering process. For the soliton to escape from the well, these vibrations must be in the right phase when the soliton tries to climb out of the well. This is illustrated by the fact that the critical velocity is a periodic function of $L$ (Fig [7).

To verify this point, we have presented 2 models based on a pseudo-geodesic approximation to the full process. Both models approximately describe the evolution of the soliton in the well and describe some of its vibrational modes. We have found that to describe the scattering of the soliton on the well reasonably accurately the effective models must take into account at least 2 vibrational modes of the soliton, one of which corresponds to a vibrational mode that radiates.

We have thus shown that the scattering of solitons on a well has interesting properties that need to be investigated further. Many other models similar to the NBS model can also have wells and it would be interesting to find out if they exhibit similar phenomena to the ones that we have described in this paper and if the vibrational modes of the solitons always play such a crucial role.

\section{Acknowledgement}

This investigation is a natural follow up of the work [5] performed in collaboration with Joachim Brand. We would like to thank him for this collaboration. The work reported in this paper was, in part, supported by a PPARC grant (PPA/G/S/2003/00161).

\section{References}

[1] P G Kevrekidis, Integrability revisited: a necessary condition. Phys Lett A 285 (2001) 383-389.

[2] B.M.A.G.Piette and R.S. Ward, Physica D 201, 45-55 (2005)

[3] Z. Fei,, Yu.S. Kivshar, and L. Vazquez, Phys. Rev. A 45, 6019-6030 (1992)

[4] R.H. Goodman and R. Haberman, Physica D 195, 303-323 (2004).

[5] B.M.A.G. Piette, W.J. Zakrzewski and J. Brand, J. Phys. A 38, 1-10 (2005)

[6] T Weidig, The baby skyrme models and their multi-skyrmions. Nonlinearity 12 (1999) 1489-1503. 